\title{
Cas historique d'améloblastome mandibulaire géant
}

\section{RÉSUMÉ}

Aida BENHAMMOU

Service d'O.R.L.

et de chirurgie maxillo-faciale,

Hôpital des spécialités,

C.H.U. Rabat-Salé, Maroc,

Avenue El Araar, Résidence Ribat El Fath,

Appt E1, 10100 Hay Ryad, Rabat,

Maroc.

\section{Malik BOULAADAS}

Service d'O.R.L.

et de chirurgie maxillo-faciale,

Hôpital des spécialités,

C.H.U. Rabat-Salé, Maroc.

\section{Farah HAJJ}

Service d'O.R.L.

et de chirurgie maxillo-faciale,

Hôpital des spécialités,

C.H.U. Rabat-Salé, Maroc.

Leila ESSAKALI

Service d'O.R.L.

et de chirurgie maxillo-faciale,

Hôpital des spécialités,

C.H.U. Rabat-Salé, Maroc.

\section{Mohamed KZADRI}

Service d'O.R.L.

et de chirurgie maxillo-faciale,

Hôpital des spécialités,

C.H.U. Rabat-Salé, Maroc.

L'améloblastome est une tumeur bénigne odontogène, atteignant le plus souvent la mandibule. Le diagnostic est généralement fait à I'occasion de problèmes dentaires ou devant l'apparition d'une tuméfaction mandibulaire. Son traitement est chirurgical, et l'exérèse complète ne doit pas empêcher une surveillance régulière du fait du risque de récidive.

Les auteurs rapportent un cas historique d'améloblastome mandibulaire géant, diagnostiqué à un stade tardif du fait du retard de consultation, ayant bénéficié d'une exérèse complète et reconstruction secondaire par un lambeau libre microanastomosé de péroné.

améloblastome

- mandibule

chirurgie

- reconstruction

- lambeaux libres
AOS 2011;253:39-43

DOI: $10.1051 /$ aos/2011104

(C) AEOS / EDP Sciences 


\section{Introduction}

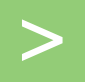

L'améloblastome est une tumeur bénigne, localement agressive, à fort potentiel de récidive, dont l'origine se situe au niveau de l'épithélium odontogène. II s'agit de la plus fréquente des tumeurs odontogènes, puisqu'elle en représente 9 à $11 \%$. La mandibule est atteinte dans plus de $80 \%$ des cas.

Les formes géantes, dues au retard de consultation, sont fréquentes dans les pays en voie de

\section{Observation}

Notre patient était un jeune homme d'origine mauritanienne, âgé de 26 ans, sans antécédents pathologiques notables, admis pour la prise en charge d'une volumineuse tumeur mandibulaire évoluant depuis sept ans et longtemps négligée par le patient.

L'examen retrouvait un patient en bon état général, eupnéique, présentant une énorme développement et sont responsables de déformations faciales importantes, posant des problèmes de prise en charge.

Nous rapportons un cas d'améloblastome mandibulaire géant chez un jeune patient ayant bénéficié $d^{\prime}$ 'une exérèse chirurgicale et d'une reconstruction par un lambeau libre de péroné, et discutons des différentes possibilités de reconstruction après exérèse de ce type de tumeur.

tuméfaction de la région mandibulaire droite, mesurant $23 \mathrm{~cm}$ dans son grand axe, indolore, de consistance variable (tantôt rénitente, tantôt dure), polylobée et fixée par rapport aux deux plans, occupant une partie importante de la face et de la cavité buccale empêchant l'occlusion buccale, sans signes inflammatoires en regard (fig. 1 a et 1 b).
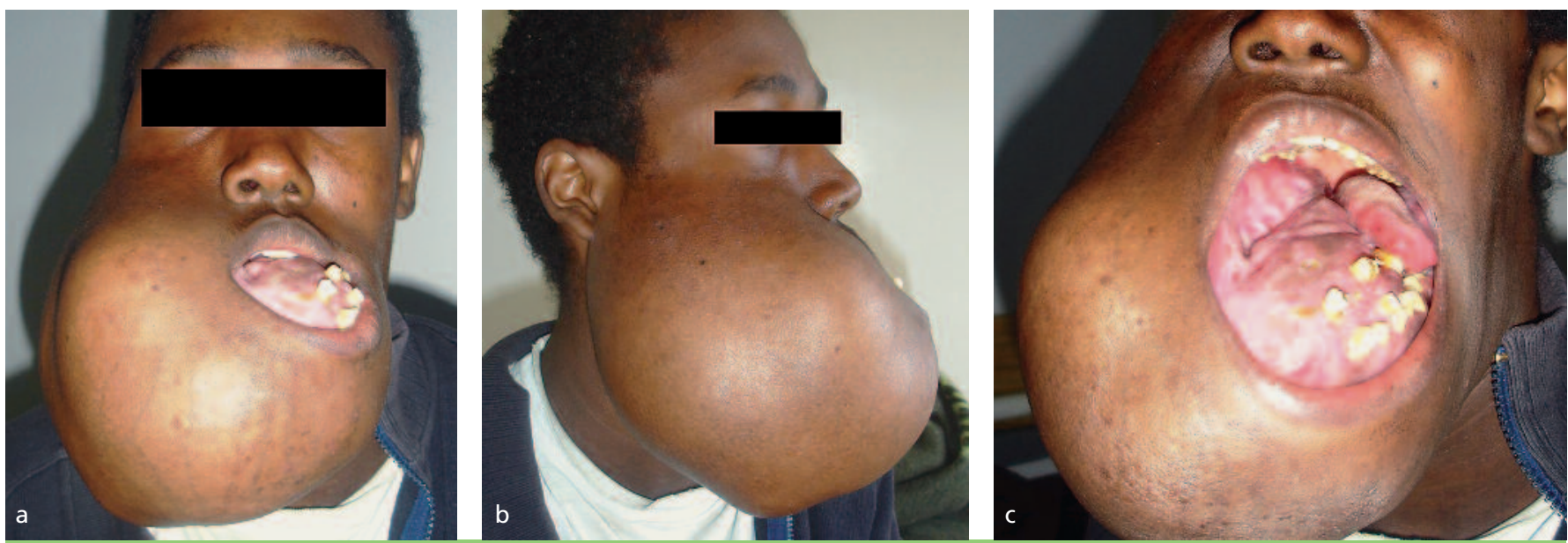

Fig. 1 a à c Vue de face (a), de profil (b), et endobuccale (c) montrant la volumineuse tuméfaction mandibulaire, exerçant un effet de masse sur les structures endobuccales. 
En endobuccal, l'expression tumorale était très importante, avec bombement pelvien et avulsions dentaires (fig. $1 \mathrm{c}$ ), sans limitation d'ouverture buccale ni ulcération muqueuse. L'examen cervical n'objectivait pas d'adénopathies palpables.

Le bilan scannographique réalisé a mis en évidence une volumineuse tumeur mandibulaire hétérogène, polykystique, cloisonnée avec soufflure osseuse et lyse corticale par endroits, sans signes de malignité (fig. 2). Le diagnostic d'améloblastome était fortement suspecté. L'intervention chirurgicale a consisté en une exérèse tumorale complète et radicale. II s'agissait d'une hémimandibulectomie droite avec désarticulation homolatérale, élargie jusqu'à l'angle mandibulaire gauche (fig. 3). L'étude anatomopathologique était en faveur d'un améloblastome bénin, dont les limites d'exérèse étaient saines. Les suites opératoires étaient simples.

Six mois après l'intervention, le patient a bénéficié d'une reconstruction mandibulaire par lambeau libre microanastomosé de péroné (fig. 4). Les résultats esthétiques et fonctionnels sont satisfaisants.

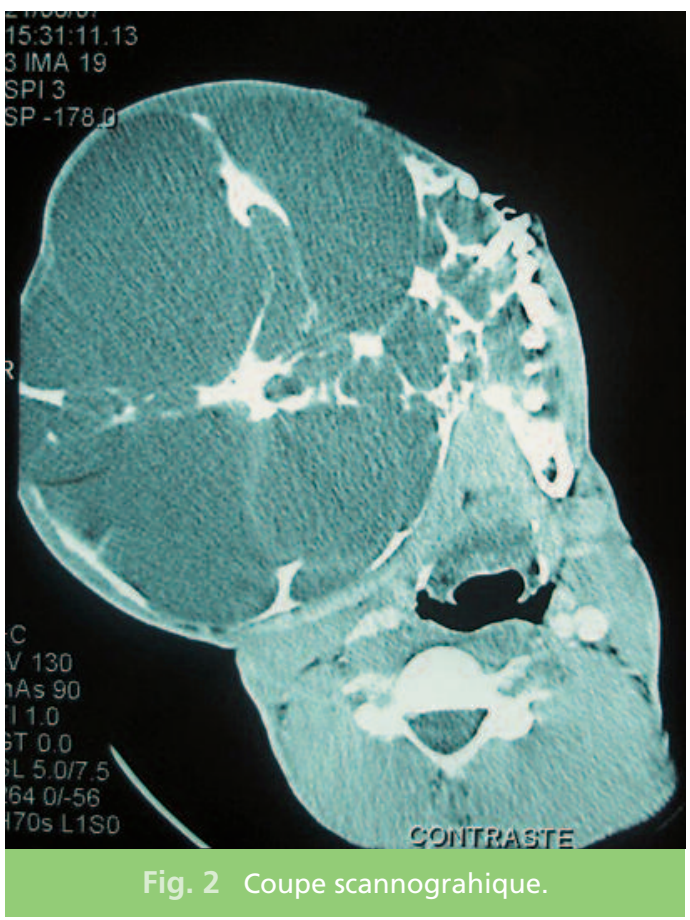

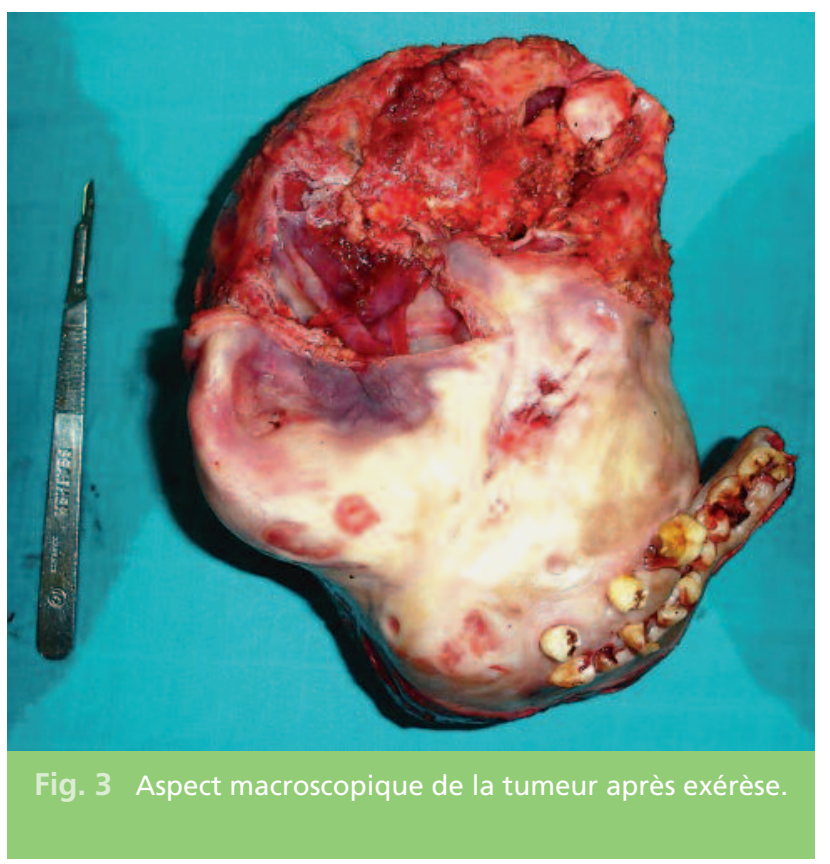

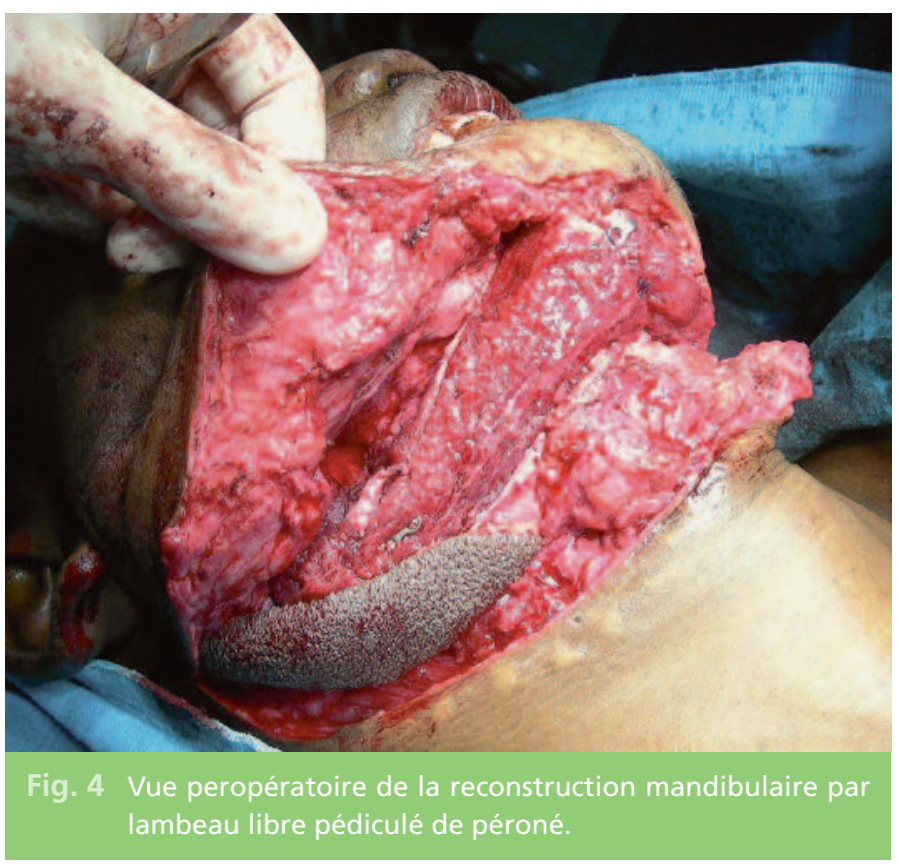




\section{Discussion}

L'améloblastome est la plus fréquente des tumeurs odontogènes, atteignant la mandibule dans I'immense majorité des cas. À ce niveau, la branche montante est la plus fréquemment touchée, dans $70 \%$ des cas, suivie de la région prémolaire dans $20 \%$ des cas, et enfin de la région symphysaire dans les $10 \%$ restants. Elle se voit chez le sujet jeune, entre 30 et 50 ans, sans prédominance de sexe [1].

Ce type de tumeur se caractérise, dans les pays en voie de développement, par son volume souvent important, chez des patients à l'hygiène bucco-dentaire défectueuse [1].

Cliniquement, l'évolution est lente avec soufflure osseuse progressive. L'améloblastome est souvent asymptomatique, de découverte fortuite lors de la réalisation de radiographies standards, pour une pathologie dentaire le plus souvent. Sinon, les principaux motifs de consultation sont représentés par une tuméfaction gingivale, des douleurs faciales ou dentaires, des mobilités dentaires, ou encore des troubles parodontologiques. Tardivement peuvent apparaître une déformation jugale, une névralgie ou même une anesthésie du nerf dentaire inférieur [2].

Radiologiquement, l'image typique de l'améloblastome est polygéodique, faite de géodes franches, homogènes, arrondies ou ovalaires, à contours nets et réguliers, séparées par des ponts osseux et disposées sans ordre réalisant l'image classique en "bulles de savon ". À un stade plus évolué, tout cloisonnement peut disparaître. Des dents peuvent être scellées dans la tumeur ou à côté d'elle [1, 2].
Sur le plan histologique, on distingue trois types d'améloblastome : folliculaire, plexiforme ou mixte.

Le traitement radical consiste en une exérèse chirurgicale large avec des marges saines. La reconstruction dans le même temps doit être recommandée en raison du taux élevé de patients perdus de vue. De plus, les conséquences d'une amputation mandibulaire large sont non négligeables, aussi bien sur le plan esthétique que fonctionnel (sur la mastication, la déglutition et la phonation) [1, 3]. Les lambeaux libres osseux microanastomosés représentent actuellement le traitement de choix de ces pertes de substance mandibulaire, le site donneur préférentiel est le péroné $[3,4]$.

Des greffons osseux non vascularisés sont proposés par quelques auteurs, mais pour des pertes de substances modérées inférieures à $9 \mathrm{~cm}$ de longueur $[1,4]$.

L'évolution est marquée par la récidive souvent tardive, d'où l'intérêt d'une surveillance rigoureuse et prolongée, à la fois clinique et radiologique. Les formes polykystiques et folliculaires seraient plus pourvoyeuses de récidive. Ceci impose une exérèse d'emblée complète et le contrôle histopathologique systématique de toute tumeur mandibulaire, car le taux de récidive est étroitement lié au traitement chirurgical effectué [1, 3].

Une dégénérescence maligne surviendrait dans $5 \%$ des cas, sous forme de carcinome ou, plus rarement, de sarcome. De rares cas de métastases régionales ou à distance ont également été rapportés [1]. 


\section{Conclusion}

L'améloblastome est une tumeur bénigne polymorphe, localement invasive, caractérisée par sa tendance à l'extension et à la récidive.

De rares cas d'améloblastomes géants ont été déjà décrits, notre cas est I'un des plus volumineux, diagnostiqué à un stade avancé. La chirurgie à ce stade est mutilante d'où la nécessité d'un temps de réparation. La surveillance reste indispensable mais illusoire en milieu sousmédicalisé.

La présentation de ce cas géant d'améloblastome nous rappelle les difficultés d'accès aux soins et de suivi des malades dans les pays en voie de développement.

\section{Bibliographie}

1. Abbas $A$, Renaux $A$, Pochan Y, Faye A, Sissoko B. Améloblastome géant de la mandibule. Rev Stomatol Chir Maxillofac 2006;107:171-3.

2. Kalavrezos N, Baldwin DJ, Walker DM.

Giant neeglected ameloblastoma: single stage treatment and clinicopathological review.

Br J Oral Maxillofac Surg 2008;46(7):591-3.

3. Hughes CA, Wilson WR, Olding $M$.

Giant ameloblastoma: report of an extreme case and a description of its treatment. Ear Nose Throat J
1999;78:568-74.

4. Zemann $\mathrm{W}$, Feichtinger $\mathrm{M}$, Kowatsch E, Karcher H. Extensive ameloblastoma of the jaws: surgical management and immediate reconstruction using microvascular flaps. Oral Surg Oral Med Oral Pathol Oral Radiol Endod 2007;103:190-6.

\section{SUMMARY}

\section{Historic case of giant mandibular ameloblastoma}

Aida BENHAMMOU,

Malik BOULAADAS,

Farah HAJJ,

Leila ESSAKALI

Mohamed KZADRI

\section{Keywords \\ - ameloblastoma \\ - mandible \\ - surgery \\ - recontsruction \\ - free flaps}

Ameloblastoma is a benign odontogenic tumour, arising in the mandible in $80 \%$ of the cases. The diagnosis is generally easy; symptoms are essentially dental abnormalities or mandibular tumefaction. The treatment is based on surgery, the resection has to be complete and the follow-up is very important because of the high risk of recurrence.

The authors report an historic case of giant ameloblastoma of the mandible, diagnosed at a very late stage. The tumour was removed by segmental mandibulectomy, and the reconstruction was made at a second time, with a fibular free flap. 
Pour s'abonner, appeler le :

tél. + 33 (0)1 69181514 - fax + 33 (0)1 69860678

France et Union Européenne version papier + version électronique

TTC

$213 €$

Reste du monde

version papier + version électronique $243 €$

Étudiant* : France et Union Européenne

(* sur présentation d'une attestation)

version papier + version électronique $129 €$

Étudiant* : Reste du monde

(* sur présentation d'une attestation)

version papier + version électronique

Membre de I'AEOS

Prix au numéro

(*) Offre valable jusqu'au 31/10/2011 $183 €$

$67 €$
4 NUMÉROS ${ }^{*}$ )

TTC

version électronique seule $186 €$

version électronique seule $156 €$

version électronique seule $114 €$

$147 € \quad$ version électronique seule $116 €$

Frais de port au numéro

$4 €$

Je souhaite $\mathbf{m}$ 'abonner à la Revue AOS

$\square$ Je souhaite prolonger mon abonnement à la Revue AOS

\section{MODE DE PAIEMENT :}

- Paiement effectué par chèque à l'ordre de : EDP Sciences - REVUE AOS

17 avenue du Hoggar - P.A. de Courtabœuf

B.P. 112 - 91944 Les Ulis Cedex A (France)

Tél. + 33 (0)169181514 - Fax + 33 (0)1 69860678

e-mail : abonnements@edpsciences.org

- Paiement en ligne disponible sur notre site www.aos-journal.org
Paiement par carte bancaire :
Carte Visa
$\square$ Master Card
American Express

$N^{\circ}$

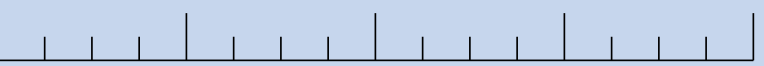

Noter ici les 3 derniers chiffres situés au dos de la carte

Date d'expiration

\section{Signature obligatoire}

\section{VOTRE ADRESSE :}

Vous êtes: $\square$ Chirurgien-Dentiste $\square$ Stomatologue $\square$ Orthodontiste $\square$ Autre: précisez Nom :

Prénom :

Adresse :

Code Postal Ville

Tél Fax E-mail 